\title{
L'edizione del “Tristan et Lancelot”. Ovvero: sull'utilità e il danno delle edizioni critiche
}

\section{Francesco Benozzo}

\section{(2) OpenEdition}

Journals

Edizione digitale

URL: http://journals.openedition.org/studifrancesi/8451

DOI: 10.4000/studifrancesi.8451

ISSN: 2421-5856

Editore

Rosenberg \& Sellier

Edizione cartacea

Data di pubblicazione: 1 décembre 2008

Paginazione: 596-602

ISSN: 0039-2944

Notizia bibliografica digitale

Francesco Benozzo, «L'edizione del "Tristan et Lancelot". Ovvero: sull'utilità e il danno delle edizioni critiche», Studi Francesi [Online], 156 (LII | III) | 2008, online dal 30 novembre 2015, consultato il 12 janvier 2021. URL: http://journals.openedition.org/studifrancesi/8451 ; DOI: https://doi.org/10.4000/ studifrancesi.8451

\section{(c) (i) (2)}

Studi Francesi è distribuita con Licenza Creative Commons Attribuzione - Non commerciale - Non opere derivate 4.0 Internazionale. 


\title{
L'edizione del "Tristan et Lancelot". Ovvero: sull'utilità e il danno delle edizioni critiche
}

\author{
A Clemente Mazzotta \\ in memoriam
}

\section{Parte prima: sull'utilità e il danno delle edizioni critiche}

Comincio questo mio intervento, rovesciando il titolo che gli ho voluto dare, con una confessione franca: se non fosse per il ricordo di due bei giorni trascorsi a Torino lo scorso febbraio, nel trasogno di cose solo mie, e per l'amabile ospitalità delle organizzatrici del colloquio sul nostro Pierre Sala, non avrei accettato di spedire un mio contributo per questi atti congressuali. La ragione è semplice: mentre ha sicuramente avuto un significato discutere in quell'occasione con i dottorandi (o comunque davanti a loro) dei criteri editoriali che ho adottato nella mia edizione del Tristan et Lancelot ${ }^{1}$, non ha invece alcun senso, dal mio punto di vista, riproporre le stesse riflessioni in questa sede, oltretutto assai prestigiosa, che ospita gli atti del colloquio in questione. La mia edizione è infatti uscita da poco meno di dieci anni, alcuni illustri recensori l'hanno misurata e valutata, con alcuni di loro ho già dialogato a distanza a proposito delle mie scelte editoriali: da questo punto di vista io dovrei forzare, e non poco, me stesso e la mia idea di ricerca ${ }^{2}$ parlando per l'ennesima volta di questo capitolo di ecdotica e variantistica d'autore.

Ma poiché l'occasione fa l'uomo ladro, e poiché anche in questa versione a stampa del mio intervento vorrei rivolgermi in particolare ai dottorandi e ai giovani studiosi, io mi permetto in questo caso di "rubare" le pagine che mi si mettono a disposizione per esporre alcune mie opinioni sulla filologia d'oggi, partendo proprio da Pierre Sala, e in particolare da un fatto che a mio giudizio dovrebbe provocare un certo senso di allarme in tutti quelli che fanno il nostro mestiere: l'annuncio, risalente a qualche tempo fa, di una nuova edizione del romanzo di cui dovrei di nuovo parlarvi, imminente per i tipi Champion di Parigi ${ }^{3}$. Nella conclusione di un mio libro recente sulla cosiddetta tradizione trobadorica ho parlato della filologia come di una disciplina da tempo «agonizzante» ${ }^{4}$ : il fatto che una nuova edizione critica del Tristan et Lancelot stia per arrivare sugli scaffali dei nostri dipartimenti costituisce un'ulteriore clamorosa conferma di questa mia convinzione. Parliamoci chiaro, come guardandoci negli occhi: ognuno di noi sa e sente dentro di sé che un'operazione editoriale di questo tipo sarebbe inutile anche se si fosse scovato da qualche parte un

(1) Cfr. P. SALA, Tristan et Lancelot, edizione critica a cura di F.B., Alessandria, Edizioni dell'Orso, 2001; sul titolo, cfr. F. BENOzZO, A proposito del titolo di un romanzo arturiano di Pierre Sala su Tristano e Lancillotto, «Quaderni di Filologia romanza della Facoltà di Lettere e Filosofia dell'Università di Bologna», X, 1993, pp. 209-228.

(2) Che ho provato a illustrare di recente nel mio Etnofilologia, «Ecdotica», IV, 2007, pp. 208-230.

(3) Ne sarà curatrice C. VERCHÈRE, della quale si veda per adesso "En ceste tribulation comme au dormir de la mort". Le "Tristan" de Pierre Sala, in Le roman français au XVI siècle, ou le renouveau d'un genre dans le contexte européen, Presses Universitaires de Strasbourg, 2005.

(4) F. Benozzo, Cartografie occitaniche. Approssimazione alla poesia dei trovatori, Napoli, Liguori, 2008, p. 59; sulla stessa linea, cfr. anche ID, La tradizione smarrita. Le origini non scritte delle letterature romanze, Roma, Viella, 2007 e ID., Back to the Hidden Cave. Ethnophilology of the European Tradition, Roma, Viella, 2008. 
terzo manoscritto, anche se avessimo la certezza che esso è autografo, e anche se questo codice contenesse delle chiose di Rabelais o Ronsard. Allo stesso modo, come ho avuto modo di scrivere e di riaffermare in più occasioni pubbliche, ritengo che non abbia avuto un gran significato che io preparassi, a suo tempo, una nuova edizione, se non forse per il fatto di avere introdotto e utilizzato i principi della «fenomenologia dell'originale» ${ }^{5}$ in un settore che vi era parso fino a quel momento refrattario. Al di là di questo, tutti noi sappiamo come vanno queste cose: a uno studente del dottorato si deve pur affidare l'edizione di qualcosa, e al termine di quegli anni di apprendistato e di studio si fa in genere in modo che questa tesi esca in forma di libro, specie se lo studente in questione ha suo malgrado incominciato a manifestare l'intenzione di fare i propri tentativi per restare tra le mura del mondo accademico. Resto però convinto - e la cosa riguarda in primo luogo, a scanso di equivoci, me stesso - che tra l'ammettere la natura seriale e in fin dei conti concorsuale di questa procedura e il confonderla con ciò che dovrebbe essere la ricerca (anche quella filologica) c'è di mezzo un mare che, per come la vedo io, assomiglia a un oceano piuttosto vasto. E allora dico questo: passi (con riserva) una seconda edizione del testo di Sala a distanza di quasi 45 anni dalla prima, che è ormai fuori commercio ${ }^{6}$. Ma se il glorioso editore Champion si è ridotto a stampare una terza edizione, a sette anni dalla precedente, non dico della Commedia di Dante (e anche qui avrei le mie belle perplessità) ${ }^{7}$ ma di questo romanzo arturiano tardivo, di un autore che è certamente simpatico a chiunque se ne sia in qualche modo occupato, ma che non possiamo per questo spacciare come meritevole di tutta questa attenzione editoriale, vuole senz'altro dire - se mi si passa il ricorso a un'espressione corrente - che "siamo arrivati alla frutta".

Davvero, però, vorrei che avessimo la capacità di tornare a farci delle domande importanti. Quella che mi viene in mente in questo caso è: «A chi serve (esclusa ovviamente, per le ragioni che ho detto, colei che ne sarà la curatrice) una nuova edizione del Tristan et Lancelot?». Non sto ingenuamente parlando del mondo reale extra-filologico, al quale come sappiamo non serve nemmeno l'edizione critica della Bibbia, ma proprio di noi filologi romanzi e francesisti. Siccome l'edizione in questione non è ancora apparsa, nell'attesa (che confesso non essere fremente) provo a rispondere parlando della mia, la quale

1) potrebbe essere servita - come ha scritto Gilles Roques - perché in qualche modo «renouvelle l'intérêt que l'on porte à Pierre Sala»;

2) potrebbe essere servita per animare un confronto sulle tecniche di edizione: è quello che è accaduto tra David Expert e me sulla «Revue critique de philologie romane», dove ci siamo confrontati a distanza su due diversi modi di leggere (e quindi editare) il testo 9 , a mio parere non comprensibile al di fuori di una filologia di tipo autoriale e di un'edizione di tipo stratigrafico-variantistico ${ }^{10}$;

(5) Cfr. D’A.S. Avalle, Principî di critica testuale, Padova, Antenore, 1978, pp. 33-43.

(6) Cfr. P. SAla, Tristan. Roman d'aventures $d u$ xvie siècle, ed. L. MuIr, Genève-Paris, Droz-Minard, 1958.

(7) Che condivido con l'amico Marco Veglia, autore di quello che a mio parere è il contributo ecdotico più importante degli ultimi trent'anni sulla Commedia, nel quale viene tra l'altro ribadita - non solo tra le righe - la fondamentale inutilità di tentativi editoriali globali se l'unico risultato che questi producono risulta alla fine essere quello di promuovere a testo qualche variante lessicale al po- sto di qualche altra: cfr. Sul testo della "Commedia" (da Casella a Sanguineti), «Studi e problemi di critica testuale», LXVI, 2003, pp. 65-119.

(8) G. Roques, Compte rendu de P. SALA, Tristan et Lancelot, ed. Benozzo, «Revue de Linguistique Romane», LXVII, 2003, pp. 292-294, a p. 294.

(9) Cfr. D. EXPERT, Compte rendu de P. SALA, Tristan et Lancelot, ed. Benozzo, «Revue critique de philologie romane», IV-V, 2003-2004, pp. 21-30; F. BenOzZO, Risposta, ibid., pp. 32-36.

(10) Oltre che alle pp. 87-111 dell'edizione in questione, e oltre alle considerazioni che avrò modo di esporre più avanti, cfr. i miei Fenomenologia 
3) potrebbe essere servita per i linguisti che si occupano dell'evoluzione degli usi grafici nel primo Cinquecento, nel caso che abbiano perso tempo a dare un'occhiata alle 40 pagine di tavole che ho pubblicato in appendice, dedicate proprio all'evoluzione delle grafie nel passaggio dal manoscritto di Aberystwyth a quello bodmeriano;

4) potrebbe essere servita per rendere accessibile un testo che esisteva soltanto in un'edizione Droz-Minard del 1958; ma anche qui potremmo legittimamente chiederci: «rendere accessibile a chi?». Dovrei forse girare questa domanda alle Edizioni dell'Orso, dalle quali in ogni caso posso garantire di non avere ricevuto in questi anni diritti d'autore tali da farmi pensare a un ardente interesse suscitato da questa pubblicazione.

Non mi pare che la mia edizione possa essere servita a molto altro - sempre ammesso (e io stesso ci credo poco) che i quattro punti elencati godano di una qualche verosimiglianza -, tanto è vero che quasi tutti gli articoli su Sala apparsi in anni successivi alla sua pubblicazione continuano a citare il testo secondo l'edizione precedente, curata da Lynette Muir, senza che questo abbia stravolto o fatto fraintendere alcunché del nostro autore. Per quei pochi che si occupano di Sala dal punto di vista storico-letterario, insomma, un'edizione vale l'altra, e io credo che questo sia un fatto del tutto normale, del quale solo qualche studioso incurabilmente affetto da quel «filologismo caricaturale» di cui parlava Contini potrebbe scandalizzarsi (o far finta di scandalizzarsi). Ebbene, se la mia edizione è servita a così poco, c'è sul serio da chiedersi a cosa servirà una terza fatica editoriale, e magari ci si potrebbe anche domandare che cosa avrebbe pensato Sala di tutti questi sforzi per consegnare alle stampe un romanzo per il quale egli aveva accuratamente e con ogni mezzo evitato, come d'altronde era accaduto per tutte le sue opere, di dare luogo a una qualsivoglia versione stampata.

Voi capite, se non altro dal tono, che la mia domanda vuole andare al di là dell'autore lionese a cui dedichiamo questo numero di «Studi Francesi». Essa riguarda un modo di essere filologi che purtroppo è ormai tipico anche delle giovani generazioni, alle quali non par vero di poter ripubblicare testi, di ridiscutere scelte editoriali precedenti, di riprodurre senza ritegno procedure ecdotiche per arrivare a tracciare i tanto agognati stemmata codicum che fanno belli e 《rendono scientifici» ${ }^{11}$ i nostri lavori. La cosa diventa sul serio fastidiosa quando costoro rispondono a chi osa criticare certi eccessi di filologismo con la frase, appresa a memoria all'epoca del loro tirocinio e ripetuta a mo' di slogan - quasi un ordine di scuderia -, che «l'ecdotica è interpretazione». Altro che interpretazione, filologi cari! Per quanto mi riguarda (ma conversando con alcuni colleghi - di cui non farò i nomi - mi pare qualche volta di non essere l'unica mosca bianca) la nuova edizione di un testo già precedentemente pubblicato ha eventualmente un senso soltanto quando introduce, ribadisce e sancisce un principio generale nuovo. Ad esempio ritengo che praticamente tutte le edizioni dei trovatori andrebbero rifatte, riconoscendo finalmente che il metodo lachmanniano utilizzato per ricostruire i presunti testi originali di questi poeti non ha alcun senso, e introducendo una prassi editoriale più credibile, attenta alla storia

dell'originale di un romanzo cortese: la situazione manoscritta del "Tristan et Lancelot", «Francofonia», XXIX, 1995, pp. 19-48 e La vicenda testuale del "Tristan et Lancelot". Critica delle varianti e filologia arturiana d'autore, «Studi mediolatini e volgari», LXXII, 1997, pp. 47-66.

(11) L'espressione è stata usata a più riprese, pro- prio con riferimento alla possibilità di ricostruire lo stemma codicum, da Cesare Segre in occasione di un recente seminario interdottorale organizzato dalla Scuola Superiore di Studi Umanistici su "Il metodo delle scienze umane" (Bologna, 8 maggio 2008). 
delle singole canzoni e capace di considerarle appunto come "canzoni" e non come "testi". Così come andrebbero rifatte molte edizioni di chansons de geste, per le quali lo stemma codicum non è che un miraggio privo di significato ${ }^{12}$. Ma assistere all'uscita, nel corso degli anni, di due, tre, quattro, cinque edizioni critiche praticamente identiche, siano esse di Chrétien de Troyes, di qualche autore minore antico-francese, di Dante, di Pierre Sala, di Montaigne o di Shakespeare, è un fatto scoraggiante che alla fine sembra dare ragione a chi riconosce nella filologia una disciplina ormai inutile e ingombrante, che è finita per diventare, da strumento che era o doveva essere, un autentico fine autoreferenziale. È abbastanza noto, in questo senso, il giudizio espresso da Giovanni Testori su Contini: «La filologia è stata per lui una vera mania: invece di servirsene come di un mezzo opportuno, ha finito spesso per gettarla sull'opera come una coltre» ${ }^{13}$.

Ma va detto con chiarezza anche questo: di un grande maestro come Contini si potrà dire tutto il bene e tutto il male che si vuole senza che la sua statura venga sul serio toccata. Quello che invece preoccupa - spero non solo me - è la proliferante crescita del «giardino dei finti Contini» ${ }^{14}$, ai cui abitatori sembra che non resti altro da fare, al momento, che editare, sezionare e vivisezionare i testi di cui dovrebbero invece nutrirsi. Più che umili, fedeli e scrupolosi restauratori di affreschi, molti di loro assomigliano ormai ad addetti che montano le impalcature a ridosso degli stessi, il cui unico risultato è di fatto quello di impedire una fruizione dell'opera di cui si dichiarano interpreti e custodi ${ }^{15}$.

\section{Parte seconda: l'edizione del "Tristan et Lancelot"}

Ma a questo punto mi voglio fermare e, per non sottrarre me stesso da quello che è in fondo un compito che mi è stato assegnato, torno a parlare dei criteri editoriali che hanno guidato la mia edizione, cercando di far tesoro di alcune osservazioni emerse dalla giornata di studio torinese.

Il Tristan et Lancelot è tradito, come la quasi totalità delle opere di Sala, da due manoscritti: A [Aberystwyth, Llyfrgell Genedlaethol Cymru, 443-D] e B [Cologny, Bibliothèque Bodmer, 148]. L'analisi grafica dei codici indica $\mathbf{B}$ come esemplare più elegante, caratterizzato da un ductus posato e regolare. Anche l'analisi della campitura e delle miniature conferma l'impressione che $\mathbf{B}$ possa essere identificato nella copia d'offerta, quella destinata a far circolare il romanzo negli ambienti della corte di Francesco I, destinatario dell'opera. Questa ipotesi è comprovata da un'analisi mi-

(12) Cfr. il mio Filologia al bivio: ecdotica celtica e romanza a confronto, «Ecdotica», I, 2004, pp. 2454 , dove elenco alcune notevoli operazioni editoriali nell'ambito della filologia celtica, in grado di confermare e mettere alla prova alcune ipotesi critiche. Simile al caso delle chansons de geste è quello rappresentato dai cantari, per il quale suggerisco vivamente la lettura di C. DONÀ, Cantari, fiabe e filologi, in Il cantare italiano fra folklore e letteratura, a cura di M. PICONE e L. RubinI, Firenze, Olschki, 2007, pp. 147-170.

(13) Cit. da L. Doninelui, Conversazioni con Testori, Parma, Guanda, 1993, p. 102.

(14) Ho sentito per la prima volta questa espressione dalla voce di Clemente Mazzotta, in occasione di una delle sue lezioni di filologia italiana. Stando alla testimonianza di Luciano Formisano, Contini stesso ebbe sentore del proliferare incontrollato di questi seguaci e della conseguente storpiatura di un metodo che egli aveva propugnato per difendere una certa idea di testo e di tradizione: mi riferisco «agli eccessi di certa microfilologia applicata ai testi minori, se non anche a certo esasperato formalismo di cui in qualche modo si riteneva responsabile, per non averlo saputo "fermare in tempo" »: cfr. L. FORMISANO, Ricordo di Gianfranco Contini professore, in Recensioni e biografie. Libri e maestri. Atti del $2^{\circ}$ seminario, Alghero, 19-20 maggio 2006, a cura di P. MANINCHEDDA, Cagliari, CUEC, 2007, pp. 19-37, a p. 37.

(15) Massimo Sannelli parla, a questo proposito, di «filologia-gabbia»: cfr. la sua Introduzione ad AlANI DE InSUlIS, Anticlaudianus, a cura di M.S., Trento, La Finestra, 2004, p. 21. 
crolinguistica e grafica dei due testimoni, dalla quale emerge con evidenza l'esistenza di serie «irreversibili» ${ }^{16}$ di fenomeni che danno conto di un'evoluzione cosciente e coerente nel passaggio da un esemplare all'altro. In questo senso, B, codice seriore, mostra anche nella grafia una fisionomia più recente, che tende ad adeguarsi da un lato al dato fonetico e dall'altro alle norme codificate dai grammatici del primo Cinquecento $^{17}$. Questa fenomenologia grafica trova conferma nell'analisi dei rapporti più propriamente testuali tra i due manoscritti: meno curato nell'aspetto, A mostra infatti talune lezioni depennate, insieme ad altre aggiunte in interlinea, e si configura come una sorta di codice preparatorio, come una stesura dell'opera che registra in sé anche quelle correzioni, quei ripensamenti e quegli aggiustamenti che documentano il lavoro dell'autore nel passaggio dalle fonti al proprio romanzo ${ }^{18}$.

Un aspetto importante per le conseguenze che esso assume nelle scelte editoriali è rappresentato dagli indizi di autografia dell'opera: oltre ai fatti che ho elencato nell'introduzione alla mia edizione (e che qui mi limito a riassumere: 1) la grafia di entrambi i codici sembra la stessa delle Fables, considerate autografe dai precedenti editori $^{19}$; 2) alcune varianti in interlinea illustrano un processo correttorio tipico dei manoscritti d'autore; 3 ) le piccole mende mantenute anche in $\mathbf{B}$ lasciano interpretare questo esemplare come un codice che non è stato sistematicamente riletto o collazionato e si inquadrano in una tipologia di copie d'autore che ci è nota grazie a casi quali l'autografo decameroniano del Boccaccio; 4) l'epistola dedicatoria e la miniatura che apre il romanzo descrivono Sala come un umanista che scrive e trascrive di persona la propria opera ${ }^{20}$, aggiungo un fatto cruciale, emerso anche dal dibattito seguito al mio intervento torinese: che bisognerebbe cioè sfidare il buonsenso per immaginare una fenomenologia di allestimento di queste opere in duplice redazione diversa da quella dell'allestimento personale delle stesse. Nessun'altra ipotesi appare verosimile, a meno che non ci si immagini 1) che esistessero originali autografi di Sala affidati successivamente a un qualche copista, e che siano andati tutti perduti; 2) che il nostro autore dettasse l'opera a un qualche copista (mentre numerosi errori dimostrano che siamo di fronte a trascrizioni di fonti scritte). Mi sembra di poter dire che tutti i relatori intervenuti alla giornata di studio di Torino abbiano convenuto con me su questo punto. Per tutte queste ragioni, è dunque evidente che la tradizione in cui collocare i due manoscritti del Tristan et Lancelot va identificata in una tradizione d'autore, e che la metodologia ecdotica da utilizzare è quella della fenomenologia dell'originale.

Per riepilogare, A è il manoscritto anteriore, l'esemplare personale dell'autore, una specie di codice degli abbozzi che reca anche testimonianze della rielaborazione che l'autore vi ha portato. B, più elegante, è l'apografo di A, l'ultimo stadio licenziato dall'autore. Le sue lezioni divergenti sono di quattro tipi: a) errori di copia, b) emendamenti di lezioni palesemente erronee, c) integrazioni di lacune, d) varianti graficofonetiche, morfofonetiche, lessicali e sintattiche. Nei suoi rapporti con A, il codice bodmeriano rappresenta insomma qualcosa di simile a ciò che, rispetto alla redazione a lui vicina, rappresenta l'autografo berlinese Hamilton 90 per la tradizione del De-

(16) Nel senso di D. IsELla, Le carte mescolate. Esperienze di filologia d'autore, Padova, Liviana, 1987, p. 109.

(17) Si tratta delle serie ou $\mathbf{A}>o \mathbf{B}$, ez $\mathbf{A}>$ es $\mathbf{B}$, $c \mathbf{A}>s \mathbf{B}, i(n) c t \mathbf{A}>i(n) t \mathbf{B}$, ung $\mathbf{A}>$ un $\mathbf{B}, p p \mathbf{A}>p$ $\mathbf{B}$ (in protonia), $i \mathbf{A}>y \mathbf{B}$.

(18) Trovo inutile tornare a elencare gli esempi che danno sostanza a questa affermazione: se ne veda l'elenco e l'analisi in P. SALA, Tristan et Lancelot, ed. Benozzo, cit., pp. 81-84.
(19) Cfr. A. Forni MarmocchI, Un'opera inedita di Pierre Sala; "Fables et emblèmes en vers", "Atti dell'Accademia delle Scienze dell'Istituto di Bologna, Classe di Scienze Morali. Rendiconti», LXIII, 1975, pp. 149-187; EAD., Un nuovo manoscritto delle "Fables" di Pierre Sala (con sette favole inedite), ibid., LXVI, 1978, pp. 1-35, a p. 35.

(20) Bibliografia e riferimenti specifici in P. SALA, Tristan et Lancelot, ed. Benozzo, pp. 84-85. 
cameron: «non è un manoscritto privato, di servizio, su cui l'autore abbia lavorato proprio come autore [...] ma è copia materiale di quel codice privato e di servizio [...]. L'autore, cioè, è, sì, evidentemente presente nella trascrizione [...] ma presente come trascrittore e non come scrittore [...] dovette preoccuparsi specialmente della presentazione [...]: e in questo appare impegnato più come amanuense e libraio che come autore ${ }^{21}$. B appare, allora, come una versione modernizzata di A: non una reale ultima volontà dell'autore, né una vera e propria seconda fase redazionale, ma una particolare forma (più elegante e graficamente conforme alle norme grammaticali cinquecentesche) del romanzo. $\mathbf{A}$, esemplare che rimarrà nella biblioteca privata di Sala, non disdegna, quasi a indicare una propensione nostalgica ${ }^{22}$, una patina linguistica arcaica, mentre $\mathbf{B}$, trascritto per l'ambiente moderno e modernizzante della corte, si adegua anche linguisticamente alle nuove tendenze cinquecentesche. Questa conclusione è confortata anche da uno studio delle varianti «sostitutive» - in senso continiano - di $\mathbf{B}$ rispetto ad $\mathbf{A}$, che nella mia edizione riconduco alla seguente fenomenologia: a) espunzioni e omissioni, b) semplificazioni nell'ordine sintattico, c) sostituzioni lessicali, d) omissioni di frasi ${ }^{23}$.

Avendo individuato questo tipo di tradizione autoriale, i criteri che ho adottato nella mia edizione sono volti a garantire tanto l'integrità formale dei manoscritti quanto il riconoscimento del processo elaborativo dell'opera. La mia scelta è stata quella di promuovere $\mathbf{B}$ a testo, dando tuttavia conto, in una fascia specifica di apparato, delle caratteristiche interne di $\mathrm{A}$. Ciascuna redazione possiede così un proprio apparato. Tali apparati (quello di B pubblicato in calce al testo, quello di $\mathrm{A}$ in appendice) registrano in primo luogo, per i rispettivi codici, le particolarità interne (depennamenti, lezioni ripristinate in interlinea, aggiunte successive), facendo ricorso a un sistema di segni diacritici - che qui non mi sembra il caso di illustrare - sperimentato in particolare dalla filologia italian $\mathrm{a}^{24}$. Pur nel criterio conservativo adottato non mi sono sentito esentato dall'intervenire, nei casi di più palese necessità, con emendamenti, integrazioni ed espunzioni: dei quali depennamenti do puntuale segnalazione negli apparati. Poiché il mio scopo è stato quello di fornire un'edizione stratigrafica-variantistica, ho creduto utile fornire, in una seconda fascia d'apparato, il quadro riassuntivo di evoluzione $\mathbf{A}>\mathbf{B}$, limitatamente alle varianti lessicali, morfologiche e sintattiche, mentre in appendice ho pubblicato - come già ricordato - una tavola evolutiva di tutte le varianti grafiche riscontrabili nel passaggio da A a B. la struttura della mia edizione è pertanto rappresentabile come segue:

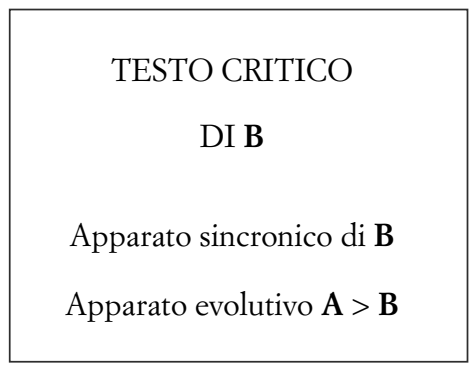

(21) V. BRANCA - P.G. Ricci, Un autografo del "Decameron" (codice Hamitoniano 90), Firenze, Olschki, 1962, pp. 36-37.

(22) Cfr. in questo senso l'analisi del romanzo di Sala nel contesto della coeva letteratura cavalleresca, così come viene abbozzata nel mio La nostalgia cavalleresca e il mito del cavaliere. Considerazioni sulla stagione rinascimentale europea delle storie di re Artù,
«L'immagine riflessa», VI, 1997, pp. 349-362.

(23) Cfr. P. SAla, Tristan et Lancelot, ed. Benozzo, cit., pp. 97-96.

(24) Cfr. soprattutto D. Isella, Le carte mescolate, cit., pp. 37-89 e C. MAzzotTA, Introduzione a VitTorio Alfieri, Panegirico di Plinio a Trajano. Parigi sbastigliato. Le Mosche e l'Api, edizione critica per cura di C.M., Bologna, Clueb, 1990. 
Nello scioglimento delle abbreviazioni, nell'introduzione di accenti, nelle distinzioni grafiche $u / v$ e $i / j$, nell'introduzione di cediglia, apostrofi e maiuscole ho seguito l'uso editoriale di sempre. ${ }^{25}$

Come ho ribadito alla giornata di studi di Torino, credo che questo metodo di edizione possa valere per la maggior parte delle opere di Sala, la cui situazione manoscritta sembra illustrare lo stesso tipo di fenomenologia autoriale ${ }^{26}$.

\section{Parte terza: conclusione}

«Concludere, voi direte, concludere che cosa? Volevo solo dire: aprire un'interrogazione, unica per tutti noi dopo che qualcosa è tornato alla luce del sole [...] per tutti gli uomini che si sono ritrovati nella buona volontà oltre che nella natura della propria ricerca» $\gg^{27}$. Non starò a ripetere cose già dette. Soltanto, invece, prenderò a prestito l'eloquente osservazione di un geomorfologo inglese: «Ecco che cosa continuarono a fare, per oltre due secoli, gli studiosi dei flussi di marea: misurarono la lunghezza delle onde e l'altezza del dislivello. E dopo averli misurati li misurarono ancora. E uscirono libri che contenevano misurazioni e discussioni sulle misurazioni. E altri libri che contenevano confutazioni delle misurazioni, e che modificavano di qualche centimetro le precedenti stime. Ci volle lo spirito di qualche osservatore meno dogmatico e più libero per farla finita con questo metodo ripetitivo. La scoperta più importante, che le maree sono governate dalla luna, si deve a questo spirito libero, non certo ai misuratori e ai confutatori di misurazioni» ${ }^{28}$.

FRANCESCO BENOZZO

(25) Cfr. M. RoQues, Étabilissement de règles pratiques pour l'édition des anciens textes français et provençaux, «Romania», LII, 1926, pp. 243-249 e A. Foulet - M.B. SPEer, On Editing Old French Texts, Arward, The Reagent Press of Kansas, 1979. Approfitto di questo intervento per segnalare, in una specie di "postuma" errata corrige, alcuni errori e sviste presenti nel testo da me pubblicato, indicando per ciascuno di essi quella che dovrebbe essere (stata) la corretta trascrizione: II.66 qui $>q u^{\prime} i$, III.28 qui > qu'i, III.205 nomme > nommé, III.303 trent $>$ trente, IV.30 aisi > ainsi, IV.73, V.104 les > ses, V.68 je n'ay > j'en ay, VI.115 alora $>$ alors, VI.149 qui > qu'i, VI.196 le > les, VII.113 les $>$ ses, VII.128 qui > qu'i,VII.11 delle > celle, VIII.125 malvastié > maulvastié, IX.85 affoyble > affoyblé, IX.99 donne > donné, IX.109 voulioit > voulloit, IX.110 faure $>$ faire, IX.123 soit ressemble $>$ soit qui ressemble, IX.131 sil nous $>$ sil qui nous, IX.172 donne $>$ donné, X. 33 arsa $>$ arse, X.87 sçavoit $>$ sçavoir, X.99 er $>$ et, X.145 ssaige $>$ saige, $\mathrm{X} .173$ conuenant $>$ convenant, X.187 aisés $>$ aises, XI.15 fontain $>$ fontaine, XI.72 no $>$ non, XI.128 $l e>l a$, XI.205 adevnture $>$ adventure, XII.10 setoit $>$ s'etoit, XIII.185 Jauray > J'auray, XIII.210 su > $d u$, XIII.255 affoyble $>$ affoyblé, XIV.25 dejurnarent $>$ sejournarent, XIV.68 forsetz $>$ forestz, XIV.73 baulter $>$ baulteur. XIV.78 lentendent $>$ l'entendent, XV.117 affoyble > affoyblé, XV.124 chapils > chaplis, XV.150 empsechera > empeschera, XV.219 maty $>$ mary, XV.362 vous > voys, XVI.360 qui > qu'i, XVI.425 lul > nul, XVI.444 myemme $>$ myenne, XVII.28 quane $>$ quant, XVII.64 vous vous $>$ vous, nous, XVIII.181 query > guery, XVIII.216 estout $>$ estoit, XVIII.314 recouvree > recouvrer, XIX.314 desha $>$ desja, XX.68 a'assirent $>$ s'assirent, XXI.25 quec > avec, XXI.38 chevale > cheval, XXI.104 dire $>$ sire, XXI.149 per $>$ par, XXI.151 facites $>$ faictes, XXI.198 emtre $>$ entre, XXII.12 por $>$ pour, XXIII.40 hoeir $>$ beoir, XXIII.41 nla $>$ la, XXIII.92 Amrc > Marc, XXIV.105 avoitr > avoir, XXIV.127 gradieux $>$ gracieux, XXIV.210 qui $>$ qu'i, XXIV.211 donnerau une aabade $>$ donnerai une aubade, XXIV.221 remps > temps, XXIV.229 de > se, XXIV.298 de > se, XXIV.339 la > le, XXIV.362 qui $>$ qu'i, XXIV.472 corant > courant, XXV.186 apres $>$ aprés, XXV.201 si > s'i, XXV.201 nen > n'en, XXV.219 donne > donné; ringrazio GILLES RoQUES e DAVID EXPERT per alcune di queste segnalazioni.

(26) Cfr. l'elenco alle pp. 32-33 della mia edizione.

(27) P. Bigongiari, Studi, Firenze, Vallecchi, 1946 [rist. Trento, La Finestra, 2004], p. 271.

(28) J. PETHICK, An Introductiuon to Coastal Geomophology, London - New York - Sydney - Auckland, Edward Arnold, 1984, p. 44. 